\title{
Enhancing Cellular Multicast Performance Using Ad Hoc Networks
}

\author{
Jun Cheol Park and Sneha Kumar Kasera \\ School of Computing \\ University of Utah \\ Email: \{jcpark, kasera\}@cs.utah.edu
}

\begin{abstract}
Although multicast communication is well-suited to shared wireless links, receiver heterogeneity impedes the use of multicast in wireless networks. In this paper, we examine an approach that addresses the receiver heterogeneity problem in cellular multicast with the help of an additional IEEE 802.11 ad hoc network. The basic idea is to allow the cellular receivers experiencing poor channel conditions to use the ad hoc network to connect to those cellular receivers that are experiencing good cellular channel conditions. The good receivers (called proxies) relay multicast data to the poor receivers through the ad hoc network.

We specifically consider the third generation cellular high data rate (HDR) Broadcast/Multicast Services (BCMCS). We develop a new routing algorithm to find efficient ad hoc paths from the proxies to the cellular multicast receivers. Unlike existing algorithms [1], our routing algorithm considers the effect of ad hoc path interference. Using simulations of an HDR BCMCS network in conjunction with an IEEE ad hoc network, we show that our algorithm improves the receiver goodput by up to $280 \%$ compared to that obtained without using ad hoc paths. We also show that our algorithm achieves up to $98 \%$ higher receiver goodput in comparison to the greedy algorithm proposed in [1].
\end{abstract}

\section{INTRODUCTION}

The growing dependence of important social, financial, medical, and defense applications and services on networked systems and the demand for making these available ubiquitously are fueling a tremendous growth of mobile systems and cellular wireless networks. However, although the cellular wireless bandwidth is growing with the deployment of newer air interface technologies, it is still a scarce and precious resource. For applications where multiple wireless users receive the same content, the use of multicast will help in preserving wireless bandwidth.

Multicast is an efficient paradigm for transmitting packets from a single sender to multiple receivers [2]. In wireless networks, due to the shared nature of the wireless links, multicast transmission instead of multiple individual unicasts of the same data to multiple receivers is more efficient. We envision several scenarios that could benefit from cellular multicast. Earlier research has identified information dissemination (e.g., news updates, stock quote updates), distance learning, shared whiteboards [3] and networked virtual environments etc. as important applications that could benefit from multicast capability. In addition to the above applications, we believe that, in emergency scenarios such as a natural or man made disaster where wireless resources are likely to be heavily overloaded, efficient multicast capability could play a significant role in providing emergency communication while better utilizing the wireless bandwidth.

Receiver heterogeneity is one of key impediments in using multicast for wireless networks ${ }^{1}$. Receivers using a wireless link encounter different channel conditions making it hard for a multicast sender to transmit at a rate that is suitable for all the receivers. The receiver heterogeneity problem becomes worse in the case of mobile and wireless networks because of the mobility of receivers, that results in dynamic changes in the ambient conditions.

In this paper we tackle the problem of efficient multicast in cellular wireless networks. In order to address the problem of receiver heterogeneity in efficient wireless multicast transmission, we examine an approach that uses wireless 802.11-based ad hoc networks in conjunction with the cellular networks. The basic idea is to allow the cellular receivers experiencing poor cellular channel conditions to use the ad hoc networks to opportunistically connect to those cellular receivers (also called proxies) that are experiencing good cellular channel conditions. The good receivers relay multicast data to the poor receivers through the ad hoc networks. Such a use of an additional wireless network allows us to tackle the cellular wireless receiver heterogeneity problem.

The above approach of using ad hoc networks in conjunction with cellular networks was first proposed in [1] for cellular unicast ${ }^{2}$. However, we study this approach in the context of cellular multicast. We specifically consider the third generation high data rate (HDR) Broadcast/Multicast Services (BCMCS) proposed in [4]. Additionally, in finding ad hoc paths from receivers experiencing poor cellular channel conditions to the proxies (receivers experiencing good cellular channel conditions) we also consider the effect of transmission interference in the ad hoc network. This transmission interference reduces the data rate along the ad hoc paths. In order to determine the reduction in ad hoc data rates due to transmission interference, we use the minimum distance-2 vertex coloring (Min-D2Color) [5]. Min-D2-Color is a graph coloring problem that determines the minimum number of colors required to color the vertices of a graph such that no two vertices separated by two or less number of edges have the same color. Modeling

\footnotetext{
${ }^{1}$ Receiver heterogeneity is a fundamental problem in multicast for both wireless and wired networks as well.

${ }^{2}$ The term proxy is borrowed from [1].
} 


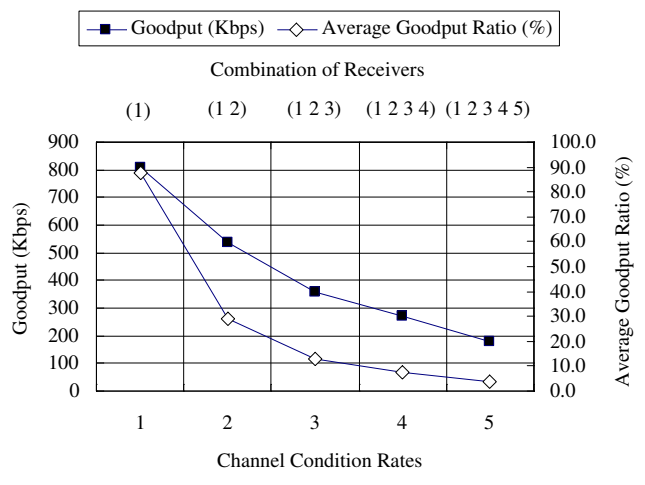

Fig. 1. Receiver heterogeneity problem

the ad hoc network as a graph with ad hoc nodes as its vertices and considering the interference across nodes to be limited to two hops, the minimum number of colors required to color the graph of ad hoc nodes such that no two nodes separated by two or fewer hops have the same color, provides the reduction in data rate. The ad hoc data rate is further reduced due to the overheads associated with scheduling the shared access channel in the ad hoc network. We also take this reduction in ad hoc data rate into account. Next, using the data rate reduction information, we develop a simple, yet efficient heuristic algorithm for determining near optimal ad hoc paths. To evaluate our algorithm, we simulate an HDR network with BCMCS support. We find that the use of Min-D2-Color allows us to choose efficient ad hoc paths and the multicast goodput improves up to $280 \%$ in comparison to the multicast goodput obtained without using ad hoc paths. We also find that our algorithm achieves up to $98 \%$ higher goodput in comparison to the greedy algorithm proposed in [1].

The remainder of the paper is organized as follows. In the next section, we motivate our research by demonstrating the receiver heterogeneity problem. In Section III we discuss the related work. The Section IV provides our problem setting and relevant background on the proposed HDR BCMCS standard. How the Min-D2-Color could be used as a model for determining the data rate reduction in ad hoc paths and how the data rate reduction is used to develop an ad hoc routing algorithm, is presented in Section V. Simulations for evaluating our approach and the results are included in Section VI. We conclude the paper in Section VII.

\section{Motivation}

One effort to overcome receiver heterogeneity problem in cellular multicast is CDMA2000 high rate broadcast/multicast packet data air interface specification by 3GPP2 [4]. It is also known as Broadcast/Multicast Services (BCMCS) which is the 3G multicast proposed standard. In BCMCS, a base station sends the multicast data at a fixed data rate to all the receivers in a multicast group for each broadcast/multicast service regardless of the various wireless channel conditions of the receivers. Since a fixed data rate is used, nodes that have lower data rates than the BCMCS served data rate may not receive all the packets. BCMCS uses a forward error correction (FEC) mechanism to increase the probability of reconstructing the original data packets correctly [4], [6]. As a result, BCMCS can achieve reliable multicast up to a certain multicast data rate regardless of the number of receivers in a multicast group. Consequently, it alleviates the receivers' heterogeneity problem to some extent.

However, as the multicast transmission rate increases, the number of nodes that cannot reconstruct all the data packets increases rapidly. Although lower data rates can be achieved in BCMCS, they are insufficient for certain multimedia applications.

To demonstrate the impact of the receiver heterogeneity problem in the context of BCMCS, we evaluate a simple scenario where a group of cellular receivers receive multicast data from a $3 \mathrm{G}$ HDR base station. We use two metrics for this evaluation - receiver goodput and average goodput ratio. Receiver goodput is defined to be the data that is correctly received by a receiver in a given time minus any redundant data. The average goodput ratio is defined to be the average goodput of all the receivers over the rate of data transmission from the base station.

Fig. 1 shows how the receiver goodput and the average goodput ratio varies with heterogeneous channel conditions. The channel conditions are labeled as numbers on the $\mathrm{x}$-axis with level 1 representing the best channel condition and level 5 representing the worst channel condition. The left y-axis shows the channel conditions for each label as the receiver goodput for receivers. The right y-axis shows how the average goodput ratio varies with the receivers experiencing channel conditions from level 1 to 5 . The five points showing the average goodput ratio curve correspond to receivers experiencing (1), $(1,2),(1,2,3),(1,2,3,4)$ and $(1,2,3,4,5)$ channel conditions. The multicast performance degrades considerably with increase in heterogeneity.

\section{RELATED WORK}

Cellular and ad hoc networks have been mostly studied independently. More recently, the integration of these two networks is getting increasing attention [1], [7], [8]. Ad hoc networks could benefit from the centralized architecture of the cellular networks. On the other hand, nodes connected to the cellular networks could benefit from the higher bandwidth of ad hoc networks and maintain connectivity among themselves during absence of access network connectivity possibly due to poor channel conditions or even equipment failure.

iCAR [7] uses ad hoc relay stations at strategic locations to achieve cell load balancing. The relay nodes in this approach are static. Our work in motivated by UCAN [1]. UCAN first proposed the use of ad hoc networks in conjunction with cellular networks for cellular unicast. It provided algorithms for finding ad hoc paths from receivers experiencing poor cellular channel conditions to those experiencing good channel conditions (proxies). Our work differs from UCAN in the following significant ways. First, we examine the use of 
the cellular network in conjunction with an ad hoc network to overcome the receiver heterogeneity problem in cellular multicast so as to improve the receiver data rates. Instead of 3G HDR unicast, our focus is on HDR BCMCS [4], [6]. Second, unlike UCAN, we can consider the interference in the ad hoc network and not just the proxy data rates in finding the appropriate ad hoc paths. We use a Min-D2-color model [5] to quantify the affects of the ad hoc network interference and develop a simple, yet efficient heuristic algorithm for finding ad hoc routing paths. In comparison to the Min-D2-color, many of the other existing graph theoretic solutions [5], [9][12] are less practical and compute intensive. Third, in our simulation we use richer models for simulating multipath fast fading.

\section{PROBLEM SETTING}

We consider a single $3 \mathrm{G}$ base station that is serving a geographical area called a cell. The cell contains several mobile nodes served by the base station. Each mobile node has two wireless networking interfaces: 3G HDR BCMCS and IEEE 802.11. When HDR BCMCS data is transmitted from the base station, some mobile nodes that experience good cellular channel conditions receive the data directly from the base stations. Others that do not experience good channel condition receive the data from the proxies through the ad hoc network. Unlike unicast transmission, IEEE 802.11 links provide simplistic mechanism for broadcast/multicast. In this work, we use only unicast transmission in ad hoc paths. The case of ad hoc multicast will be studied in a future paper.

IEEE 802.11 uses a CSMA/CA mechanism for media access control. Additionally, the use of request to send (RTS) and clear to send (CTS) messages keep in dealing with hidden terminal problems. A typical unicast communication consists of a RTS/CTS/DATA/ACK cycle. The use of RTS/CTS messages result in extra overheads which is justified only for large data packet sizes. In our work, we use RTS/CTS messages but minimize the overheads by aggregating data at the proxies so that data is forwarded in sufficiently large packets.

We now present some relevant background on BCMCS. Broadcast/Multicast Service (BCMCS) has been proposed for downlink multicast (from base station to cellular receivers) in HDR networks [4]. BCMCS uses an FEC based mechanism to reliably serve all multicast members in the presence of poor channel conditions. The FEC mechanism uses ReedSolomon codes (on top of Turbo codes) to reduce the packet error rate at receivers experiencing poor channel conditions. Multiple Reed-Solomon error control blocks are multiplexed before transmission on the physical layer. Each error control block consists of $\mathrm{N}$ byte rows and $125 \times \mathrm{M}$ byte columns. In Reed-Solomon coding, when the size of a data packet is $125 \times \mathrm{M}$ bytes and a base station needs to send $\mathrm{K}$ data packets, it constructs $\mathrm{R}$ more packets that are the error control packets using Reed-Solomon coding. The base station sends all $\mathrm{N}(=\mathrm{K}+\mathrm{R})$ packets, instead of $\mathrm{K}$ data packets. As long as a mobile node is able to receive any $\mathrm{K}$ packets from the $\mathrm{N}$ data or error control packets, it can completely reconstruct

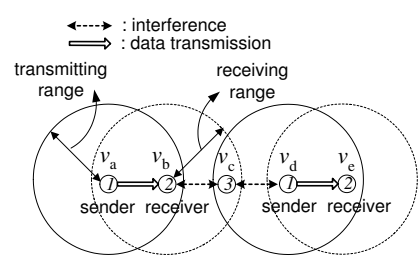

Fig. 2. Modeling ad hoc interferences

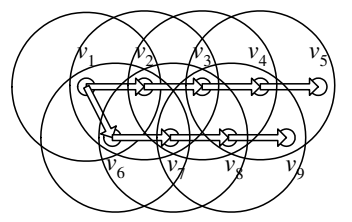

(a) Two ad hoc paths and interference ranges

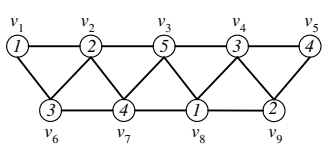
with the minimum D2-vertex coloring (b) Interference graph

Fig. 3. Two ad hoc paths with a shared starting node $v 1$ and a corresponding minimum D2-vertex coloring

$\mathrm{K}$ actual data packets. In our architecture, every proxy is responsible for reconstructing $\mathrm{K}$ data packets and forwarding the reconstructed data as one packet through IEEE 802.11 ad hoc paths. The disadvantage of using the FEC based mechanism is that the receivers experiencing good channel conditions receive redundant packets and hence suffer from loss in goodput.

\section{AD HOC ROUTING ALGORITHM}

In order to develop the ad hoc routing algorithm, we first describe an ad hoc transmission interference model in the following section. This model helps us determine the data rate reduction in the ad hoc paths due to interference Data rate is also reduced due to the overheads of IEEE 802.11 media access control. Using the data rate reduction information in the ad hoc paths due to both these factors, we develop a heuristic ad hoc routing algorithm to route data from proxies to multicast receivers through the ad hoc network.

\section{A. Ad Hoc Network Interference Model}

The understanding of data transmissions in ad hoc wireless networks is important for building an ad hoc interferences model. The unicast data transmission in IEEE 802.11 ad hoc wireless networks uses a data acknowledgment mechanism to provide reliability. As a result, for successful data transmissions, any union of a sender's transmitting range and an intended receiver's receiving range should be clear from any interference. In other words, the union of transmitting and receiving ranges is a safe transmission zone with at most one active sender and one active receiver.

Fig. 2 shows the transmitting, receiving and interference ranges. If we model the ad hoc network as a graph with ad hoc nodes as its vertices, and edges drawn between those vertices (nodes) that are within the interference range of each 


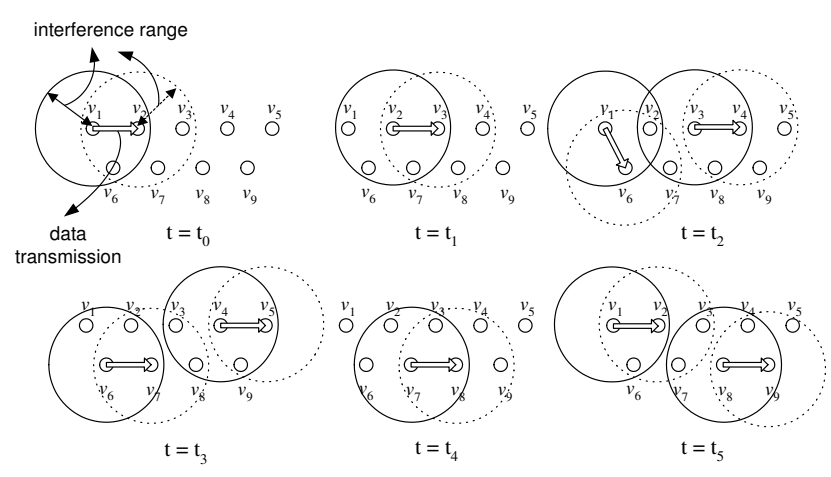

Fig. 4. One to $v_{5}$ and another to $v_{9}$

other, the interference range is limited to two edges (or two hops) in the graph. If we color the nodes of the graph such that no two vertices separated by two edges have the same color, then the minimum number of colors provide a measure of data rate reduction due to ad hoc network interferences ${ }^{3}$. This graph coloring problem is called the minimum distance-2 coloring or MIN-D2-Color problem. More formally, consider the following definition [5]. Let $G(V, E)$ be an undirected graph. A D2-vertex coloring of a graph $G(V, E)$ is defined by a mapping $f: \mathrm{V} \rightarrow\{1, \ldots, k\}$ such that $f(u) \neq f(v)$ whenever there is a path composed of at most two edges between $u$ and $v$ in $G(V, E)$. Here, $k$ is the number of colors in the mapping $f$. In the context of our networks, $G(V, E)$ is an interference graph based on the interference range of the ad hoc paths, where there is an edge $(u, v)$ if the mobile nodes $u$ and $v$ are within the interference range of each other. The MinD2-Color problem is to find a D2-vertex coloring of $G(V, E)$ with the minimum number of colors. When the graph $G(V, E)$ is constructed based on the interference range, the Min-D2Color problem of the interference graph $G(V, E)$ provides an ad hoc transmission interferences model where the union of the transmitting and receiving ranges has at most one active sender and one active receiver.

Although the Min-D2-Color problem has been used in other contexts such as the channel assignment (what is the minimum multiple channels that are needed to allow transceivers to communicate with each other without any interference [13]), and the broadcast transmission scheduling problem where the shared access channel is used for the broadcast in the ad hoc network [12], we consider this problem for data rate reduction in IEEE 802.11 ad hoc networks. Given an interference graph $G^{4}$, a solution of the Min-D2-Color problem represents the minimum data rate reduction when only a single channel is used on the ad hoc paths. We argue that the achievable data rate of each ad hoc path is reduced to at least $W / \delta_{2}(G)$ where $W$ is the data rate of an IEEE 802.11 one hop link, and $\delta_{2}(G)$ is the minimum number of colors required for distance2 vertex coloring of graph $G$. This argument is supported by the following example.

\footnotetext{
${ }^{3}$ Such an observation has also been made in [12], [13]

${ }^{4}$ Unless ambiguous, we use $G$ instead of $G(V, E)$.
}

Consider the scenario in Fig. 3(a) where there are two ad hoc paths starting from a proxy $v_{1}$ for nodes $v_{5}$ and $v_{9}$. A corresponding interference graph $G$ for this scenario is shown in Fig. 3(b) together with a solution of the Min-D2-Color problem. In the example shown in Fig. 3(b), $\delta_{2}(G)=5$.

Fig. 4 shows an optimal transmission sequence for sending data along two ad hoc paths, one from $v_{1}$ to $v_{5}$, and another from $v_{1}$ to $v_{9}$. The optimal transmission sequence of Fig. 4 . is related to the D2-vertex coloring of Fig. 3(b). For instance, while the nodes $v_{4}$ and $v_{6}$ have the same color as 3 in Fig. 3 (b), the nodes $v_{4}$ and $v_{6}$ can receive or send the data together at $t_{2}$ or $t_{3}$, respectively in Fig 4. Although the nodes $v_{1}$ and $v_{8}$ can send data at $t_{5}$ since they are colored by 1 , the node $v_{1}$ is not able to send the subsequent data to $v_{2}$ until $t_{4}$. This delays the data forwarding on the ad hoc paths by a factor of 5 , which is also the value of $\delta_{2}(G)$.

\section{B. A Simple Heuristic Method for Min-D2-Color}

The Min-D2-Color problem is NP-complete [14] not just for general graphs but even for restricted graphs [15]. However, [5], [16] observed a useful characteristic of Min-D2Color problem that makes it more usable. It can be shown that for an interference graph $G$, if maximum node degree of $G$ is $\Delta(G)$, every proper D2-vertex coloring requires between $\Delta(G)+1$ and $\operatorname{Min}\left\{(\Delta(G))^{2}+1,|V|\right\}$ colors where $|V|$ is the number of vertices. This means that a solution of Min-D2Color problem is at least $\Delta(G)+1$. In the case of Fig. 3(b), the number of colors is exactly same as $\Delta(G)+1$. We consider several examples including the one shown in Fig 8(a) and found that the minimum number of colors required for the D2-vertex coloring is $\Delta(G)+1$. More evidence is provided by the experimental results in [16] based on University of Florida Sparse Matrix Collection [17]. They study a variety of graphs and found that the minimum solutions of the D2vertex coloring are within the upper $5 \%$ of $\Delta(G)+1$ in 18 out of 22 cases (over $80 \%$ ). These observations lead us to develop the following simple heuristic method for determining $\delta_{2}(G)$ : minimum number of colors in a D2-vertex coloring of $G$ is approximated by $\{\Delta(G)+1\}$.

\section{Scheduling Overhead}

The IEEE 802.11 data rate is reduced not only due to the transmission interference, but also due to several other overheads tied to media access protocols including the use of RTS/CTS/DATA/ACK mechanism. The RTS/CTS mechanism incurs more overhead in terms of the ratio of data packet transmission time over the whole media access time. However, it becomes very effective in a congested area. This is because the probability of the transmission collisions is considerably reduced due to small size of RTS/CTS and the contention window size is prevented from growing exponentially for each transmission collision. As analyzed in [18], these overheads depend mainly on the packet size. Increasing the packet size lowers the overhead of media access protocols. In our design, we use the RTS/CTS mechanism to avoid the possible 


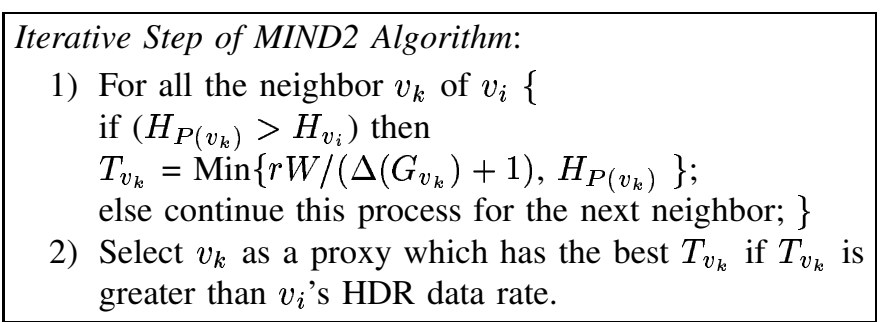

Fig. 5. Iterative Step of MIND2 algorithm

transmission collisions, and have proxies aggregate data in order to reduce the scheduling overhead.

[18] provides maximum theoretical data rates considering all the media access overheads and [19] presents extensive experimental results with various commercial $802.11 \mathrm{~b}$ wireless LANs. Their results are fairly consistent. Using either of the approaches in [18] and [19] we can predetermine the approximate reduction $r$ (where $r \leq 1$ ) in data rate due to the scheduling overhead of IEEE 802.11 for a given packet size. Thus, when we consider the data reduction over scheduling overhead, the achievable data rate of the ad hoc paths is approximated by $r W / \delta_{2}(G)$.

\section{MIND2: Proxy Routing Algorithm}

In this section we describe our proxy routing algorithm. The effective data rate that a cellular multicast network can achieve using a proxy through an ad hoc path depends on the minimum of the average HDR downlink data rate of the proxy and the achievable data rate of the ad hoc path: $\operatorname{Min}\left\{r W / \delta_{2}(G), H_{p}\right\}$ where $G$ is an interference graph of the ad hoc paths and $H_{p}$ is the average HDR data rate of a proxy $p$, and $\delta_{2}(G)$ is approximated by $\{\Delta(G)+1\}$. Our proxy routing algorithm constructs ad hoc paths from multicast receivers to proxies using this effective data rate. Since our algorithm is based on minimum distance-2 vertex coloring, we call it MIND2.

When a multicast receiver observes that its received data rate is less than the BCMCS served data rate, it sends a proxy search request to the base station. The base station probes the HDR data rates of the request initiator's neighbors while also considering the ad hoc path data rate to those neighbors. The most promising neighbor is chosen and the proxy search continues beyond this neighbor until no better proxy can be found. The iterative search step of our algorithm is described in Fig. 5. The notations used in the algorithm description are as follows.

- $v_{i}$ : an ad hoc routing initiating node

- $v_{k}$ : a neighbor node of $v_{i}$

- $P\left(v_{k}\right)$ : if a node $v_{k}$ already has a proxy, then the proxy node. Otherwise the node $v_{k}$ itself

- $G_{v_{k}}$ : a new interference graph when $v_{k}$ is assumed to be a proxy

- $H_{n}$ : the average HDR data rate of a node $n$

- $\Delta\left(G_{v_{k}}\right)$ : the maximum node degree of a new interference graph $G_{v_{k}}$

- $T_{v_{k}}$ : the effective data rate when $v_{k}$ is selected as a proxy

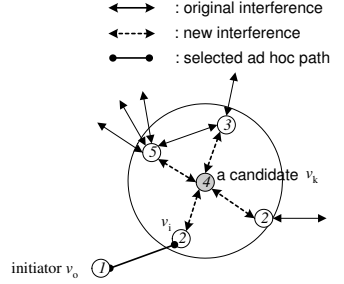

Fig. 6. Finding the maximum node degree of new interference graph

The iterative step in Fig. 5 finds a potential proxy node among the immediate neighbors. When there is a tie for $T_{v_{k}}$, a node that has smaller $\Delta\left(G_{v_{k}}\right)$ is selected as a proxy. However, it is not able to find a potential proxy if it is two or more hops away. To overcome this limitation, we enhance the iterative step to consider not only the immediate neighbors of a node but also the immediate neighbors' neighbors. Let $H 2\left(v_{k}\right)$ be a function of a node $v_{k}$. When the node $v_{k}$ already has a proxy, it returns $H_{P\left(v_{k}\right)}$. Otherwise, it returns the maximal HDR data rate among the node $v_{k}$ and its all the neighbors. The boolean condition in Fig. 5 changes to $\left(H 2\left(v_{k}\right)>H_{v_{i}}\right)$ and the statement that is executed when this condition is true changes to $T_{v_{k}}=\operatorname{Min}\left\{r W /\left(\Delta\left(G_{v_{k}}\right)+1\right), H 2\left(v_{k}\right)\right\}$. We could possibly use a larger "lookahead" but we find that our "2-lookahead", described above, is fairly efficient in finding potential proxies while also keeping the computation overhead low.

Fig. 6 shows the use of the MIND2 algorithm. In this figure, $v_{i}$ is the first proxy of an initiator node $v_{o}$. By executing the steps in Fig. 5, if $v_{k}$ is found to be a better proxy, then $v_{i}$ becomes a relay node and $v_{k}$ assumes the role of a proxy. These steps are repeated until no better proxy can be found or the HDR data rate of the proxy is greater than the BCMCS served data rate. In order to find $\Delta\left(G_{v_{k}}\right)$ as shown in Fig. 6, we add new interference edges to all the neighbors of $v_{k}$ and then find the maximum node degree (in this case, the maximum node degree is 5 ). The new data rate of ad hoc paths is then $r W /\left(\Delta\left(G_{v_{k}}\right)+1\right)$.

Note that MIND2 algorithm does not necessarily depend on the assumption that transmission range is equal to interference range because it is based on a graph that is constructed according to interference range, not transmission range.

\section{PERFORMANCE EVALUATION}

\section{A. 3G HDR BCMCS Simulation}

To evaluate our proxy routing algorithm MIND2, we simulate the 3G HDR BCMCS in the $n s-2$ simulator [20]. For an accurate simulation of the HDR downlink channel condition especially in dense urban environments, we implement Rayleigh multipath fading in the simulator [6], [21]. For the implementation of BCMCS, Reed-Solomon coding is implemented as the FEC mechanism [4]. Specifically, we use a $(\mathrm{N}=16, \mathrm{~K}=14)$ Reed-Solomon coding for evaluating our work. The cell radius is $1000 \mathrm{~m}$. We validate our simulator by comparing our simulation results with those of [6]. 


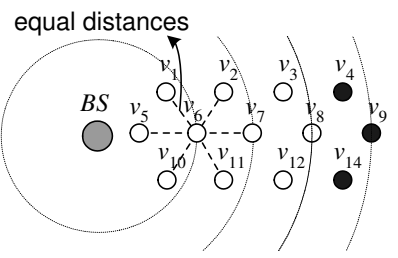

Fig. 7. An example of node distribution

\section{B. IEEE 802.11 Ad Hoc Network Simulation}

We first predetermine the value of the factor $r$. Recall from the section $\mathrm{V}-\mathrm{C}$ that $r$ is the reduction factor in ad hoc path data rates due to scheduling overheads. We use an 802.11b network whose maximum data rate is $11 \mathrm{Mbps}$ [18], [19]. In our simulation experiments, the payload size in ad hoc paths is typically around 1500 bytes based on the use of a $(\mathrm{N}=16, \mathrm{~K}=14)$ Reed-Solomon coding. Using the results of [18] and our own $n s-2$ simulation of the IEEE 802.11 media access control in the ad hoc network, we find that $r$ can be approximated fairly accurately by a value 0.4 . All ad hoc simulations are based on the Two-Ray Ground reflection propagation model [22]. Based on [22], the transmission range and interference range of a one hop link is set to $250 \mathrm{~m}$.

\section{Performance Results}

To study the effects of the transmission interference in ad hoc paths, we first experiment with a regular node distribution (within a cell) where the neighbors of a node are placed at equal distances from the node. Fig. 7 shows an example of such a node distribution. Nodes, $v_{4}, v_{9}$, and $v_{14}$, shaded black, are members of a multicast group that need to find proxies. We find these proxies using two algorithms - the greedy algorithm from UCAN [1] and our MIND2 algorithm.

Fig. 8(a) shows the three ad hoc paths that are found by the UCAN greedy algorithm that does not consider ad hoc interferences. Fig. 8(b) shows the ad hoc paths found by the MIND2 algorithm as a function of the BCMCS served data rate. Fig. 9 shows the total receiver goodput due to the use of ad hoc paths for the three cases - BCMCS, BCMCS+UCAN, BCMCS+MIND2. We find that the goodput improvement to be the highest in the case of BCMCS+MIND2. This is because for Fig. 8(a) $\delta_{2}(G)=7$ and for Fig. 8(b) $\delta_{2}(G)=5$. The interference is much higher in the ad hoc paths shown in Fig. 8(a) resulting in lower goodput when UCAN is used with BCMCS. Fig. 10 shows the improvement in MIND2's goodput over that of UCAN. For higher data rates the improvement reaches $98 \%$.

Next, for studying the effectiveness of the MIND2 algorithm in other scenarios, we consider a cell with 100 uniformly distributed nodes. We use different seeds to generate four uniform node distributions. We obtain the goodput results of Fig. 11 and Fig. 12 by averaging the goodput for each node distribution.

Fig. 11 compares the performance of using BCMCS only with that of using MIND2 in conjunction with BCMCS. The

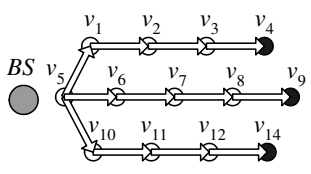

(a) Constructed ad hoc paths using a UCAN greedy algorithm

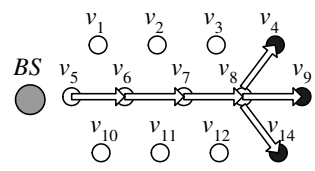

(b) Constructed ad hoc paths using MIND2 algorithm
Fig. 8. Different ad hoc paths

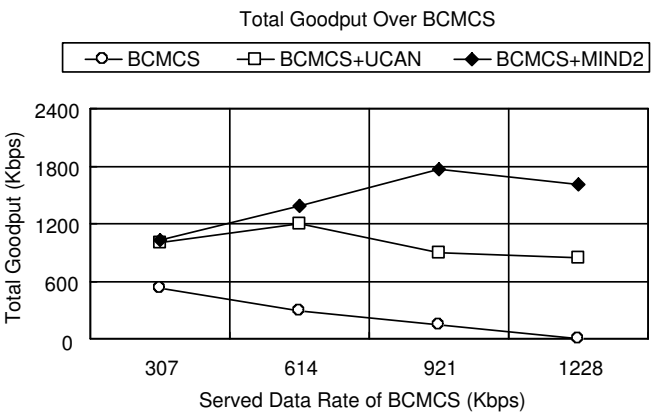

Fig. 9. Performance comparison between with and without ad hoc paths

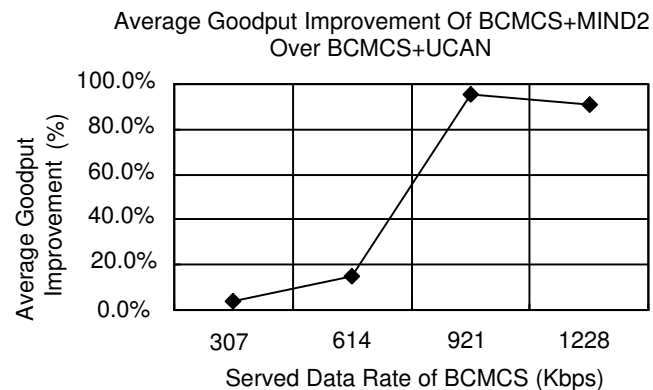

Fig. 10. Goodput gain of MIND2 over UCAN

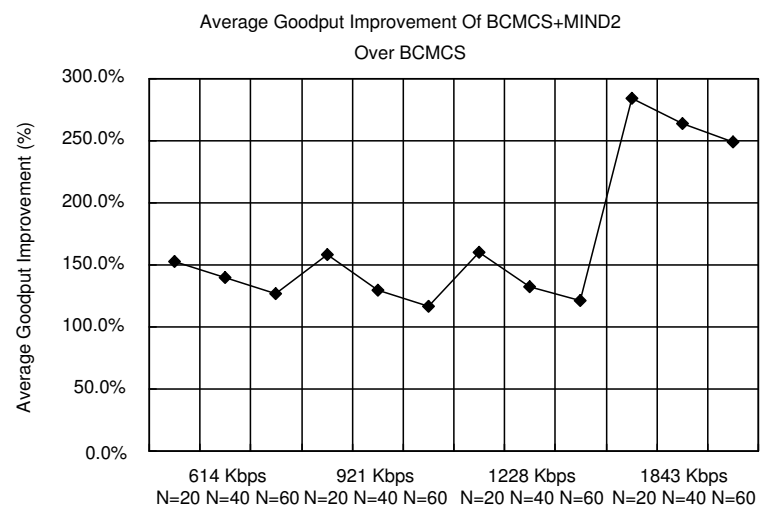

Fig. 11. Performance of ad hoc paths over BCMCS 


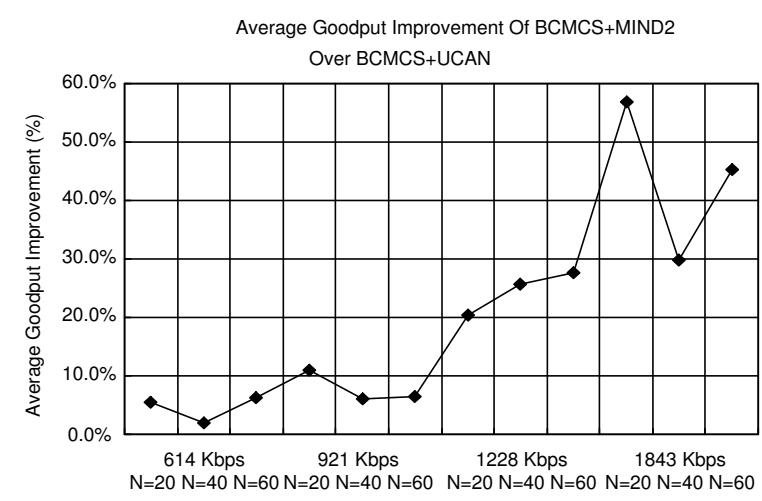

Fig. 12. Performance gain of MIND2 over UCAN algorithm

$\mathrm{y}$-axis shows the goodput improvement of BCMCS+MIND2 over BCMCS. The $\mathrm{x}$-axis represents various BCMCS served data rates with different the number of multicast receivers, $N$, among 100 nodes. With the increase in BCMCS served data rates and the number of multicast receivers, MIND2 achieves stable goodput improvement for various scenarios and up to almost $280 \%$.

Fig. 12 compares the average goodput of MIND2 with that of UCAN. This figure shows that the goodput improvement due to using MIND2 in conjunction with BCMCS increases to almost $57 \%$ over BCMCS+UCAN as the BCMCS served data rate increases. The relative goodput improvement of BCMCS+MIND2 over BCMCS+UCAN is not stable owing to the fluctuated performance of BCMCS+UCAN.

In summary, our simulations demonstrate the higher performance of BCMCS+MIND2 in comparison to BCMCS and $\mathrm{BCMCS}+\mathrm{UCAN}$ for multiple scenarios.

\section{CONCLUSION}

In this paper, we proposed a simple, yet efficient algorithm for enhancing cellular multicast performance using ad hoc networks. Our proxy routing algorithm used minimum distance-2 vertex coloring to model the effect of ad hoc interference. Using $n s-2$ simulation of $3 \mathrm{G}$ HDR BCMCS networks we found that our algorithm increases the multicast receiver goodput by up to $280 \%$ in comparison to BCMCS that does not use ad hoc paths. Additionally, we also found that our algorithm achieves up to $98 \%$ higher receiver goodput in comparison to the existing UCAN greedy algorithm [1].

Our work can proceed in several directions. We would like to study the impact of mobility on proxy selection. We also need to examine the impact of links' loss rates [23], [24]. In this paper we used only unicast communication in the ad hoc networks. We need to study the use of ad hoc multicast for improving cellular multicast performance.

\section{ACKNOWLEDGMENT}

We would like to thank Prof. Haiyun Luo of Univ. of Illinois at Urbana-Champagne for providing us the ns implementation of UCAN [1] which was the starting point of our simulations.
We would also like to thank Jae Moo Lee and Sachin Goyal for discussions of the MIND2 algorithm.

\section{REFERENCES}

[1] H. Luo, R. Ramjee, P. Sinha, L. Li, and S. Lu, "Ucan: A unified cellular and ad-hoc network architecture," in Proceedings of ACM MOBICOM, Sept. 2003, pp. 353-367.

[2] S. Deering, "Multicast routing in datagram internetwork," December 1991.

[3] S. Floyd, V. Jacobson, S. Mccanne, C. Liu, and L. Zhang, "A reliable multicast framework for light-weight sessions and application layer framing," IEEE/ACM Transactions on Networking, vol. 5, pp. 785-803, December 1997.

[4] CDMA2000 High Rate Broadcast-Multicast Packet Data Air Interface Specification, The 3rd Generation Partnership Project 2 (3GPP2) C.S0054, Feb. 2004.

[5] S. O. Krumke, M. v. Marathe, and S. Ravi, "Models and approximation algorithms for channel assignment in radio networks," in Wireless Networks, Sept. 2001, pp. 575-584.

[6] P. Agashe, R. Rezaiifar, and P. Bender, "Cdma2000 high rate broadcast packet data air interface design," in IEEE Wireless Commun. Mag., Feb. 2004, pp. 83-89.

[7] S. De, O. Tonguz, H. Wu, and C. Qiao, "Integrated cellular and ad hoc relay (icar) systems: Pushing the performance limits of conventional wireless networks," in Proceedings of the 35th Hawaii International Conference on System Sciences, 2002, pp. 3931-3938.

[8] H.-Y. Hsieh and R. Sivakumar, "On using the ad-hoc network model in cellular packet data networks," in Proceedings of ACM MOBICOM, June 2002, pp. 36-47.

[9] T. Erlebach, K. Jansen, and E. Seide, "Polynomial-time approximation schemes for geometric graphs," in Twelfth Annual Symposium on Discrete algorithms, 2001.

[10] X.-Y. Li and Y. Wang, "Simple heuristics and ptass for intersection graphs," in Wireless Ad Hoc Networks, in Dial-M'02, Sept. 2002.

[11] H. B. H. III, M. V. Marathe, V. Radhakrishnan, S. S. Ravi, D. J. Rosenkrantz, and R. E. Stearns, "Nc-approximation schemes for npand pspace-hard problems for geometric graph," Journal of Algorithms, vol. 26, pp. 238-274, 1998.

[12] S. Ramanathan and E. Lloyd, "Scheduling algorithms for multi-hop radio networks," in ACM SIGCOMM, 1992, pp. 211-222.

[13] A. Send and M. Huson, "A new model for scheduling packet radio networks," in Wireless Networks 3, 1997, pp. 71-82.

[14] S. T. McCormick, "Optimal approximation of sparse hessians and its equivalence to a graph coloring problem," in Math. Programming 26, 1983, pp. 153-171.

[15] S. Ramanathan, "Scheduling algorithms for multi-hop radio networks," Ph.D. dissertation, Department of Computer Science, University of Delaware, Newark, 1993.

[16] A. H. Gebremedhin, F. Manne, and A. Pothen, "Graph coloring in optimization revistied," Feb. 2004.

[17] T. Davis, "University of florida sparse matrix collection," June 1997, http://www.cise.ufl.edu/research/sparse/matrices.

[18] J. Jun, P. Peddabachagari, and M. Sichitiu, "Theoretical maximum throughput of ieee 802.11 and its applications," in Second IEEE International Symposium on Network Computing and Applications, Aug. 2003, pp. 249-256.

[19] A. Vasan and A. U. Shankar, "An empirical characterization of instantaneous throughput in 802.11b wlans," 2002 , http://citeseer.ist.psu.edu/vasan02empirical.html.

[20] "ns-2 simulator," http://www.isi.edu/nsnam/ns.

[21] H. Harada and R. Prasad, Simulation and Software Radio for Mobile Communications. Artech House, 2002.

[22] "Cmu monarch project," http://www.monarch.cs.cmu.edu/.

[23] D. S. J. D. Couto, D. Aguayo, J. Bicket, and R. Morris, "A highthroughput path metric for multi-hop wireless routing," in Proceedings of ACM MOBICOM, San Diego, CA, Sept. 2003, pp. 134-146.

[24] R. Draves, J. Padhye, and B. Zill, "Routing in multi-radio, multi-hop wireless mesh networks," in Proceedings of ACM MOBICOM, Sept. 2004. 